\title{
Solvent-Enhanced Transparent Stretchable Polymer Nanocomposite Electrode for Supercapacitors
}

Wei-Cheng Zhu, ${ }^{1}$ Pin-Qian He, ${ }^{1}$ Hsin-Chiao Tien, ${ }^{1}$ Hsuan-Liang Liu, ${ }^{1}$ Wen-Chang Chen, ${ }^{2}$ Weizhong $\mathrm{Lv}^{3 *}$ and Wen-Ya Lee $e^{1,4 *}$

${ }^{1}$ Department of Chemical Engineering and Biotechnology, National Taipei University of Technology, Taipei 106, Taiwan.

${ }^{2}$ Department of Chemical Engineering, National Taiwan University, Taipei 106, Taiwan.

${ }^{3}$ College of Chemistry and Environmental Engineering, Shenzhen University, Shenzhen 518060, China

${ }^{4}$ Research and Development Center for Smart Textile Technology, National Taipei University of Technology, Taipei 106, Taiwan. 


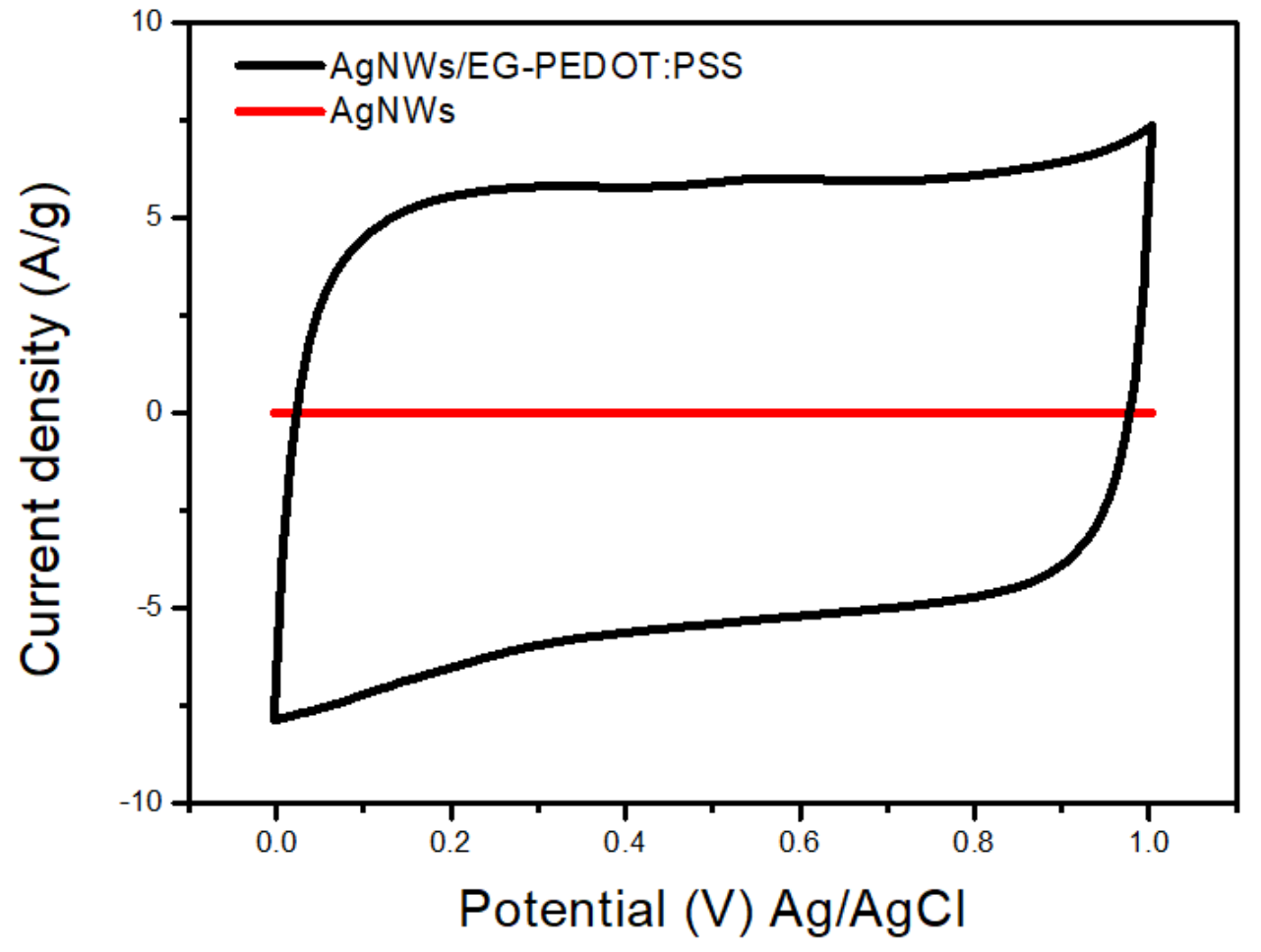

Figure S1. Cyclic voltammogram curves of AgNWs/EG-PEDOT:PSS (Black line) and AgNWonly (Red line) electrodes, respectively. The AgNW electrode without PEDOT:PSS showed negligible capacitance during cyclic voltammetry. 


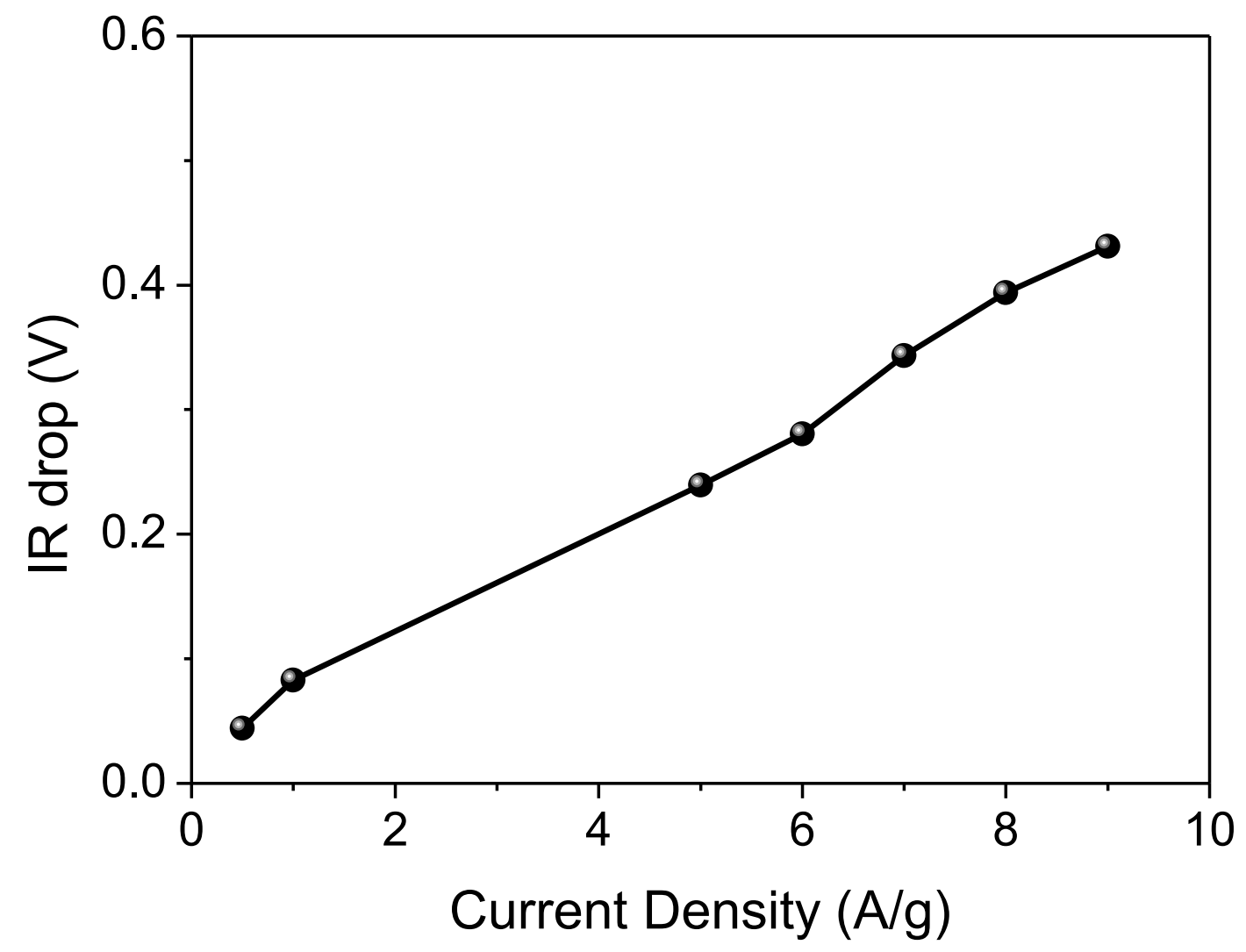

Figure S2. IR drops of AgNWs/EG-PEDOT:PSS under various current density from $0.5 \mathrm{~A} / \mathrm{g}$ to $9 \mathrm{~A} / \mathrm{g}$. 